\title{
A Rare Case of Post Traumatic Internal Fistula- A Case Report
}

\author{
Authors \\ J D Dhinesh Balaji ${ }^{1}$, T Arulappan ${ }^{2}$, K Sivaraja ${ }^{3}$, M Kishan Rao ${ }^{4}$ \\ ${ }^{1,2,3,4}$ Dept of General surgery, Sri Ramachandra institute for higher education and research, Chennai, India \\ ${ }^{1}$ Email: me.dhineshbalaji@gmail.com
}

\begin{abstract}
Internal fistulas are commonly seen following inflammatory diseases of bowel, malignancies and radiation. However, a spontaneous onset of an internal fistula following trauma is a rare presentation. We report a case of a jejuno-colic fistula following abdominal trauma.
\end{abstract}

Keywords: internal fistula, jejuno-colic fistula, post traumatic fistula.

\section{Introduction}

A fistula is an abnormal communication between two epithelialized surfaces. The fistula may be an internal fistula (entero-enteric) or an external fistula (entero-cutaneous). Primary or type I fistula develop as a result of an underlying disease affecting the gut wall. Secondary or type II fistula occur after injury to normal gut. Entero-enteric Fistulas are usually caused by inflammatory conditions such as Crohn's disease. It can also be caused by foreign bodies, radiation, diverticulitis, and malignancy. ${ }^{(1)}$

Spontaneous entero-enteric fistula following a blunt trauma abdomen is very rare and it has not been reported in literature to the best of our knowledge. This report describes a case of blunt injury abdomen causing a spontaneous Jejuno-colic Fistula, first reported to our best of knowledge.

\section{Case Report}

A 25-year-old male from presented to our hospital in June 2016 with complaints of intermittent abdominal pain for six months. Six months back, patient sustained blunt trauma to the abdomen and left arm following a road traffic accident for which he was admitted in a peripheral centre, treated conservatively for fracture of shaft of left humerus and was discharged. Pain was mild initially, becoming more severe over past four months. Patient also gave history of vomiting for 15 days, which initially contained food particles, later bilious and finally feculent content for the past 5 days.

History of loss of weight, about $20 \mathrm{~kg}$ in last 6 months. He was not a diabetic, not a hypertensive, no history of tuberculosis. On examination, patient was emaciated, pallor was present, malnourished (BMI-16.8) with muscle wasting and non-union of shaft of left humerus fracture.

Abdominal examination revealed upper abdominal distention, with a visible intestinal peristalsis (right to left) and diffuse mild abdominal tenderness. There was no palpable mass. Bowel sounds were hyperactive. Digital rectal examination was normal. Patient was hospitalized and on investigation, White blood cells-15000 cells/mm,3 haemoglobin$10 \mathrm{mg} / \mathrm{dl}, \quad$ PCV-42\%, electrolyte imbalance [potassium-3.0, sodium 116, chloride- 88, bicarbonate-26 meq/l], hypoproteinemia [total proteins -3.7, albumin-1.8] were evident. 
Ryles tube was inserted, drained feculent content. Contrast enhanced computed tomography of abdomen revealed a stricture at the level of splenic flexure cause intestinal obstruction, a fistulous communication between the splenic flexure and jejunum.

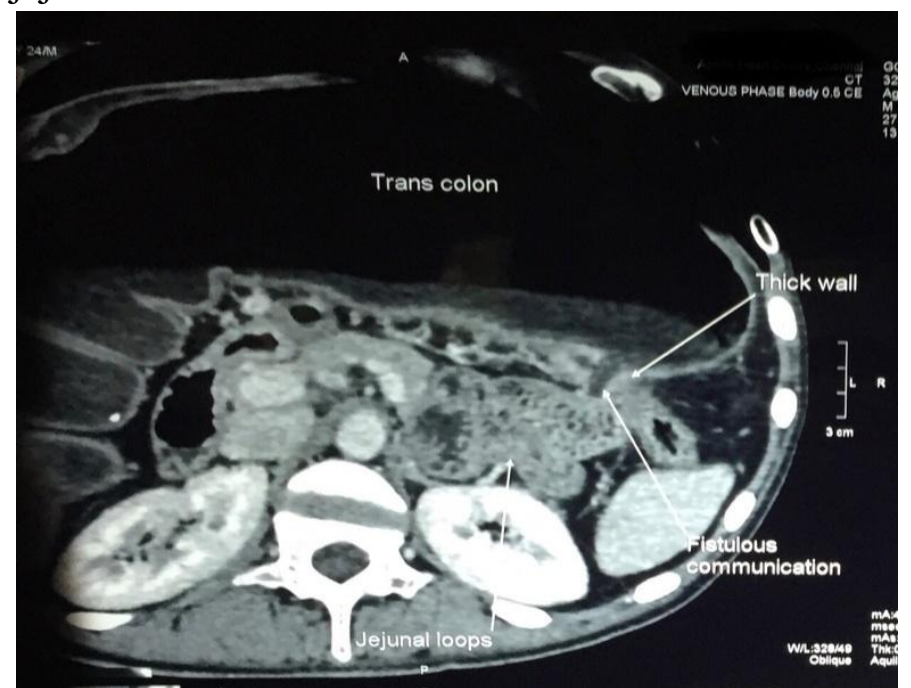

Fig 1: a contrast enhanced CT showing the fistulous tract between the jejunum and colon

Patient's general condition improved with Nasogastric aspiration, IV fluids, Antibiotics, IV albumin, total parenteral nutrition. Colonoscopy was attempted after the patient was hemodynamically stable, but the scope was not negotiated beyond the splenic flexure stricture. Patient was stabilized and taken up for surgery.

Intraoperatively, a fistulous communication was noted between transverse colon $(10 \mathrm{~cm}$ proximal to the splenic flexure) and jejunum (about $30 \mathrm{~cm}$ distal to the DJ flexure), a splenic flexure stricture was also noted. Grossly dilated transverse colon and collapsed descending colon was seen.

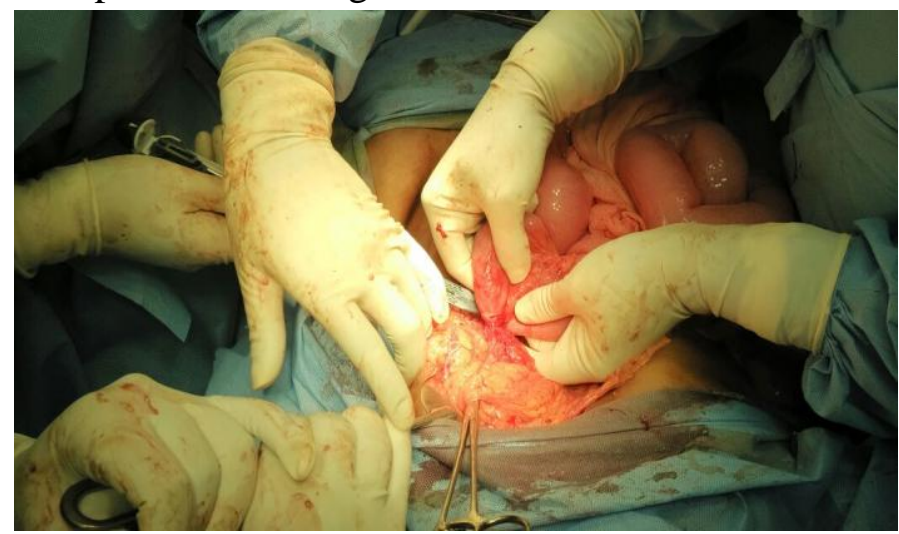

Fig 2: the fistulous tract between the jejunum and colon
Segmental resection of the stricturous and fistulous portion of colon was done and an end-to-end anastomosis was performed. Wedge resection of the jejunum with the fistulous tract was done and primarily closed. A loop ileostomy was done and the abdomen was closed. Postoperative period was uneventful. Diet was started on post-operative day 4 and the patient was discharged on post-operative day 8 .

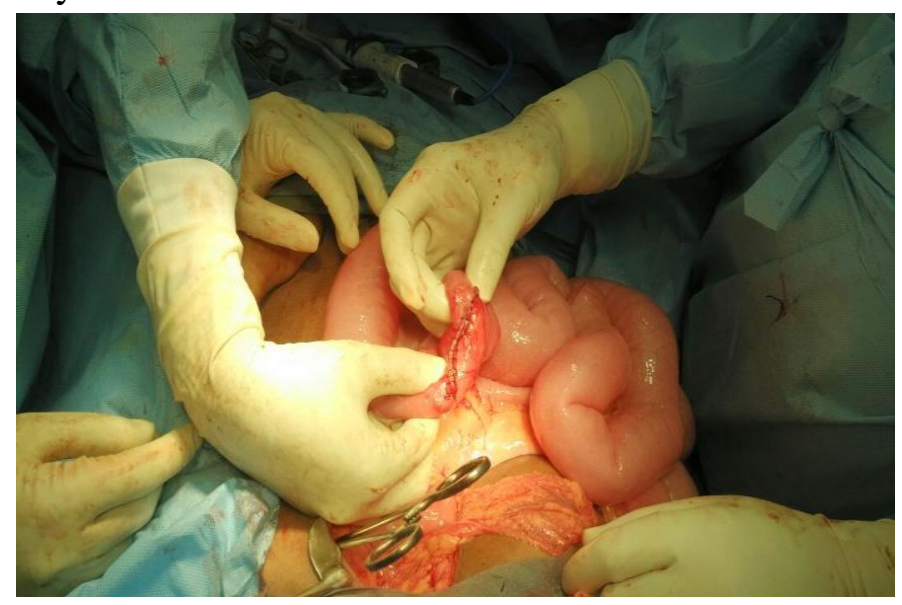

Fig 3: wedge resection of the jejunal loop and closure

Post op Histopathology: inflamed fistulous tract with no signs of malignancy/ dysplasia / granulomas. Patient gained $11 \mathrm{kgs}$ of weight after two months of follow up. Distal loopogram was done, showed normal distal colonic loops with adequate peristalsis. Hence the ileostomy was reversed.

\section{Discussion}

Most GI fistulas (75-85\%) occur as a complication of abdominal surgery. However, $15-25 \%$ of fistulas evolve spontaneously and are usually the result of intra-abdominal inflammation or infection. Fistulas that develop spontaneously without any prior iatrogenic injuries are usually manifestations of underlying malignancy, inflammatory bowel disease (I.B.D), radiation enteritis, diverticular disease, and perforated duodenal ulcers ${ }^{(1)}$. Trauma has been reported as a rare cause of GI fistulas. The mortality from entero-cutaneous fistula remains $10-30 \%$ due to complications.

Regardless of their cause, fistulas have a tremendous impact on patients and society. Increased morbidity and mortality rates, greater health care costs for diagnosis and treatment, 
prolonged hospital stays, and delayed return to work are just a few direct consequences of this condition. Gastro-jejuno colic fistulas are the most commonly seen internal fistulas following a gastrojejunostomy. Although with the advent of PPI's and developments in surgical techniques, gastro-jejunocolic fistulas can present after an inadequate vagotomy. ${ }^{(2)}$

Symptoms caused by fistulas that involve two segments of the bowel vary depending on the location of the fistula and the amount of bowel bypassed. For this reason, entero-enteric fistulas in which only a short segment of bowel is bypassed may be asymptomatic and diagnosed incidentally based on imaging findings or during surgery. however, if longer lengths of bowel are by-passed there will be significant metabolic and nutritional disturbances.

Fistulas arising from proximal bowel loops are associated with severe electrolyte imbalances and malnutrition. Fistulas arising from pancreas and ileum are least likely to close spontaneously.

Entero-vesical fistula may present as recurrent urinary tract infection (U.T.I), pneumaturia or fecaluria. Diarrhoea, abdominal tenderness, weight loss, signs of malnutrition, and electrolyte imbalances are all possible findings in patients with fistulas. ${ }^{(4)}$

Enteric fistulas follow due to inadequate blood supply to the bowel or in many situations distended or weakened small bowel due to delay in relieving partial or near total intestinal obstruction. Sometimes, following abdominal trauma a mesenteric tear can cause ischemia of bowel, followed by perforation, abscess formation, adhesion to adjacent bowel loops and finally a fistulous connection between the two loops. ${ }^{(3)}$

Malnutrition coupled with sepsis is the leading cause of mortality in GI fistulas. Blind loop syndrome, wherein there are stagnant areas in the bowel, leading to bacterial overgrowth is one of the reasons for the sepsis in a patient an internal fistula. In general principles of gastrointestinal (G.I) fistula management are divided into three phases of care; diagnosis and recognition, stabilization and investigation, and treatment and definitive care ${ }^{(6)}$ Stabilization initially concentrate on correction of fluid and electrolyte imbalance, drainage of collection, treatment of sepsis and control of fistula output (for external fistulas). The routine use of somatostatin infusion and somatostatin analogues remains controversial. Malnutrition is common, and adequate nutrition provision is essential enteral when possible, although Parenteral nutrition is often required for high output small bowel fistulae.

This is followed by investigation phase despite the fact that barium radiology is widely considered the method of choice in detection of internal fistula, accuracy was found for Ultrasonogram and computed tomography. Endoscopy is done if indicated.

Definitive surgery for fistula repair is generally delayed for several months until physiologic deficits have been restored and intra-abdominal conditions are less hostile. However, if diffuse peritonitis with ongoing sepsis is observed, immediate operative exploration may be necessary to stabilize the patient.

The preferred procedure involves excision of the fistula tract, with segmental resection of involved bowel and anastomosis of the remaining bowel. ${ }^{(5)}$ If an unexpected abscess is encountered or the quality of the bowel wall is suboptimal, a primary anastomosis is considered unsafe, instead choosing to perform a staged procedure, with exteriorization of the ends of the bowel during the first procedure. A staged repair may also be more appropriate in cases in which advanced malignancy or severe radiation changes are expected. If the procedure is performed for a malignancy, preferably, the involved segment of bowel is removed to negative margins. ${ }^{(7,8)}$

Intestinal fistulas carry high morbidity and mortality rates. If medical and non-operative treatments are not effective, the risks of surgery need to be discussed with patients and their families. Complications are common, as dense, fibrotic adhesions are likely to be encountered during surgery. Infection, bleeding, and injury to adjacent 


\section{JMSCR Vol||07||Issue||02||Page 37-40||February}

organs, as well as recurrence of the fistula, are all possibilities. To reduce these complications, patients must receive optimal nutrition.

\section{Conclusion}

Internal fistulas are more commonly associated with inflammatory bowel diseases, malignancies, radiation and previous abdominal surgeries. However, a spontaneous internal fistula following a trauma is a rare presentation. The presenting clinical picture influences the decision made in the plan of care. The timing of the definitive procedure and the plan of doing a staged procedure or single procedure varies from patient to patient. Thus, early recognition, defining the anatomy of the fistulous tract, adequate nutrition and correcting the electrolyte imbalances is the plan of management before definitive surgery. A thorough understanding of factors that influence the outcome is important.

\section{References}

1. Michael S. Nusshaum, David R. Fisher, Shackelford's Surgery of the alimentary tract $6^{\text {th }}$ edition, Chapter 73: Gastric, duodenal and small intestinal fistula Pg. no. 2720, Philadelphia, Elsevier 2007.

2. I.C Puia, C. Iancu, O Bala, et al. Gastrojejuno-colic fistula. Chirurgia, 2012. 107: $52-54$

3. Xeropotamos NS, Nousias VE, Ioannou HV, et al. Mesenteric injury after blunt abdominal trauma. Eur J Surg. 2001 feb;167(2):106-9

4. Ali Tavakkoli, Stanley W. Ashley, Michael J. Zimmer Schwartz's principles of surgery $10^{\text {th }}$ edition Chapter28: Small intestine pg. no. 1157, United states of America, McGraw-Hill Education, 2015.

5. Visschers RG, van Gemert WG, Winkens B, et al: Guided treatment improves outcome of patients with enterocutaneous fistulas. World J Surg 36:2341-2348, 2012.

6. Slade DAJ, Carlson GL. Takedown of enterocutaneous fistula and complex abdominal wall reconstruction. Surg Clin N Am 2013; 93:1163-83

7. Evenson AR, Shrikande G, Fischer JE. Abdominal abscess and enteric fistula. In: Zinner MJ, Ashley SW, eds. Maingot's abdominal operations. $11^{\text {th }}$ ed. New York: McGraw Hill; 2007: 184.

8. Fazio VW, Coutsoftides T, Steiger E. Factors influencing the outcome of treatment of small bowel cutaneous fistulas. World J Surg. 1983; 7:481. 